\section{Patient perceptions of second eye clear corneal cataract surgery using assisted topical anaesthesia}

NS Sharma ${ }^{1,2}, \mathrm{~J}-\mathrm{L} \mathrm{Ooi}^{1,2}$, EC Figueira ${ }^{1,2}$, ML Rosenberg ${ }^{1,2}, \mathrm{~K}$ Masselos ${ }^{1,2}$, DP Papalkar ${ }^{1,2}$, N Paramanathan ${ }^{1,2}$, IC Francis ${ }^{1,2,3}$, SL Alexander ${ }^{3}$ and NI Ferch ${ }^{3}$

preoperative counselling for a patient's second eye be as comprehensive as for the first eye surgery.

Eye (2008) 22, 547-550; doi:10.1038/sj.eye.6702711; published online 2 February 2007

Keywords: pain; anxiety; fear; second; topical

\section{Introduction}

Advances in cataract surgery have resulted in it being an effective and safe surgical procedure. ${ }^{1}$ General medical complications are infrequent, occurring in approximately $3 \%$ of cases, and usually limited to transient bradycardia or hypertension. ${ }^{2}$ Patient satisfaction is generally excellent; in a study of over 19000 patients undergoing cataract surgery using a variety of anaesthetic techniques, $96.9 \%$ of patients were 'very satisfied' with their pain management during and after surgery. ${ }^{3}$

Despite the impressive safety profile and visual outcomes achieved with modern cataract surgery, the experience can nevertheless engender fear and anxiety in some patients. ${ }^{4}$ It has been suggested that intraoperative visual phenomena ${ }^{5}$ experienced by patients undergoing cataract surgery with topical anaesthesia may trigger significant fear and anxiety, leading to sympathetic stimulation with hypertension, tachycardia, and possible cardiac ischaemia. ${ }^{6}$

Endocapsular phacoemulsification cataract surgery performed using local anaesthetic drops and sedation, known as assisted topical anaesthesia (ATA), has become an increasingly popular technique. ${ }^{7}$ Since our implementation of ATA several years ago, we have noted high patient acceptance; however, patients seemed

\author{
${ }^{1}$ Department of \\ Ophthalmology, Prince of \\ Wales Hospital, Randwick, \\ Sydney, Australia \\ ${ }^{2}$ The University of New \\ South Wales, Chatswood, \\ Sydney, Australia \\ ${ }^{3}$ The Ophthalmic Surgery \\ Centre, Chatswood, Sydney, \\ Australia \\ Correspondence: IC Francis, \\ Suite 12, Chatswood Grove, \\ 12-14 Malvern Avenue, \\ Chatswood, New South \\ Wales 2067, Australia \\ Tel: + 61294113277 ; \\ Fax: + 61294131839 . \\ E-mail: \\ if@student.unsw.edu.au
}

Received: 21 May 2006 Accepted in revised form: 30 November 2006 Published online: 2 February 2007

No author has a financial or proprietary interest in any methods or materials mentioned 
regularly to report they were more aware of the surgical event for the second eye compared with the first.

We determined to test our observation in a prospective, consecutive, observational, single-centre study with all operations performed by the same surgeon (ICF). Up until the past few years, surgery was performed using either retrobulbar or peribulbar blocks. ${ }^{8}$ In this study, all patients were operated using ATA, with the option of changing to assisted local anaesthesia (ALA) if necessary, utilising a retrobulbar or peribulbar block. ${ }^{9}$ We also sought to assess patient recall of intraoperative anxiety, fear, and sensory perceptions, both visual and auditory, during second eye cataract surgery using ATA in comparison with their first eye surgery.

\section{Materials and methods}

\section{Study design}

This was a prospective, consecutive, observational, single-centre study. A voluntary questionnaire was distributed to 129 consecutive patients who fulfilled the inclusion criteria. The inclusion criteria were patients undergoing first eye clear corneal cataract surgery using ATA (first surgery cohort), and those patients undergoing second eye surgery using ATA where the first eye had also been operated on using ATA (second surgery cohort). Those patients who needed to be converted to ALA or who required general anaesthesia were excluded.

\section{Questionnaire}

A questionnaire was distributed preoperatively on the day of surgery to 129 consecutive patients undergoing cataract surgery using ATA (Appendix 1). All patients agreed to participate. Patients were asked to rate intraoperative pain, anxiety, and fear using a visual analogue scale (VAS). The VAS for each symptom involved patients rating their experience of pain, fear, and anxiety from 0 (none) to 10 (worst imaginable). Recall of intraoperative visual and auditory perceptions were also questioned; these included colours, flashes, light, bright lights, shapes, music, talking, and equipment noises. Questionnaires were collected at an early postoperative visit, most commonly the day after surgery.

\section{Statistical analysis}

The distribution of both sets of data was non-Gaussian and hence results were analysed using the MannWhitney $U$ test to determine statistical significance. We used Prism ${ }^{\circledR}$ version 3. Comparisons were made between the first surgery cohort and the second surgery cohort. The Spearman correlation test was used to correlate recall of visual and auditory perceptions and combined pain, fear, and anxiety scores.

\section{Details of ATA}

Patients were operated in a free-standing dedicated ophthalmic day surgery centre. Standard cardiovascular monitoring was supervised by the anaesthetist (SLA or NIF), who explained the anaesthetic details to the patient. Small doses of intravenous midazolam, propofol, and fentanyl were titrated to individual patient need to achieve adequate relaxation yet appropriate cooperation during the case. Topical $1 \%$ amethocaine and $1 \%$ ropivacaine ${ }^{10}$ were administered after sedation.

\section{Results}

In this consecutive series, questionnaires were distributed preoperatively to 129 patients undergoing clear corneal cataract surgery using ATA. Two patients (two eyes) were converted to ALA, and were excluded from this study. This resulted in a total of 127 eligible participants.

There were 70/127 (55\%) patients undergoing first eye cataract surgery and 57/127 (45\%) undergoing second eye surgery using ATA. There were 82/127 (65\%) female patients and $45 / 127$ (35\%) male patients.

The mean pain score for the first surgery cohort was 0.80 (range $0-4$, of a reportable scale from 0 to 10 ), compared with 0.74 (range 0-5) for the second surgery cohort. There was no statistical difference between the two cohorts $(P=0.47)$.

The mean anxiety score for the first eye cohort was 1.10 (range 0-10), compared with 1.05 (range 0-8) for the second eye cohort. There was no statistical difference for intraoperative anxiety between the two cohorts $(P=0.69)$. The mean fear score was lower in the second surgery cohort compared with the first surgery cohort, with scores of 0.42 (range 0-10) and 0.63 (range 0-6), respectively, but the difference again was not statistically significant $(P=0.37)$.

Recall of intraoperative sensory perceptions showed no difference between the first and second surgery cohorts. Of a total of eight sensory perceptions studied, a mean of 3.6 was recalled in the first eye cohort compared with a mean of 3.4 in the second eye cohort. There was no statistical difference between the two cohorts $(t=0.56$, $P=0.57$ ).

There was no significant difference in pain, anxiety, or fear between male and female patients, for either the first or second eye surgery $(P$-values ranging from 0.20 to 0.79 ). 
There was an overall small but highly statistically significant positive correlation $(P=0.0002)$ between recall of intraoperative sensory perceptions and the combined fear, anxiety, and pain score (Spearman correlation coefficient, $r=0.33$; 95\% CI 0.16-0.48).

\section{Discussion}

While there have been several studies investigating patients' sensory perceptions of pain, anxiety, and fear, we believe that this is the first study to compare these perceptions in clear corneal cataract surgery using ATA, between first and second eyes. Foggitt ${ }^{11}$ studied anxiety alone in 108 patients undergoing cataract surgery. Perusal of this paper suggests that patients underwent an ophthalmic block, not ATA. A study by Habib et al ${ }^{12}$ assessed the effect of midazolam on anxiety and pain using ATA. Minimal pain and anxiety were reported, but intravenous midazolam did not seem to reduce them significantly. In this paper, second eye outcomes were not reported.

Despite our empirical observation that patients experienced more pain and sensory awareness during their second eye surgery, and which in fact led us to conduct this study, we were unable to demonstrate a statistical difference between the two cohorts. Recall of sensory perceptions during the surgery demonstrated no significant difference between the first and second eye.

We observed a small but highly statistically significant positive correlation between recall of intraoperative visual and auditory perceptions and the combined fear, anxiety, and pain score, for both first eye surgery and second eye surgery cohorts. This suggests that the ophthalmic surgeon should inform patients that they may have these sensory perceptions during surgery, whether first or second eye, and reassure them that these are normal phenomena for some patients during ATA.

Our findings contrast with previous studies. Nijkamp et $a l^{4}$ found in a qualitative study that patients reported less fear of the second eye surgery. Fagerstrom ${ }^{13}$ reported that a good result in the first eye surgery reduced the fear of the cataract operation on the second eye. Both these studies employed blocks.

Psychologists distinguish fear from anxiety. Fear is recognised as having an obvious cause and generally subsides when the stimulus is removed. By contrast, anxiety relates less clearly to a specific event and can overwhelm the patient with a chronic mindset. ${ }^{4}$ We expected that having previous experience of cataract surgery under ATA would have helped to reassure the patients and reduce their levels of fear and anxiety. Contrary to our expectations, patients in the second eye cohort did not demonstrate a statistically significant reduction in their self-reported scores for fear and anxiety.

We note that sedatives may have amnesic properties, perhaps making patient to recall intraoperative pain, fear, and anxiety less accurately. However, all the patients in the study had small titrated doses of intravenous agents to achieve similar levels of sedation, and sedation was used in both the first and second surgery cohorts.

The possible reasons for our patients demonstrating no significant difference between perceptions in their first and second eye are speculative. We expected that having been through the experience once, the patient may become more aware of some of the sensory events perceived at the first surgery. However, our study demonstrated that patients were equally aware at the second surgery. It has been suggested previously that reassurance by experienced staff may diminish anxiety in patients undergoing cataract surgery using topical anaesthesia. ${ }^{12}$ The surgeon, anaesthetist, and nursing staff may feel that the patient needs less explanation and reassurance for the second eye surgery. Our study suggests that the patient needs just as much counselling and reassurance for the second eye surgery as for the first eye.

The demonstrable lack of difference between first and second eye surgery suggests strongly that patients should be refamiliarised with the whole surgical event of their second eye. The surgeon should not presume that the patient will experience less pain, anxiety, and fear during the second operation.

\section{References}

1 Asbell PA, Dualan I, Mindel J, Ahmad M, Epstein S. Age related-cataract. Lancet 2005; 365: 599-609.

2 Schein OD, Katz J, Bass E, Tielsch JM, Lubomski LH, Feldman MA et al. The value of routine preoperative medical testing before cataract surgery. N Engl J Med 2000; 342: 168-175.

3 Katz J, Feldman M, Bass E, Lubomski LH, Tielsch JM, Petty BG et al. Injectable $v$ s topical anesthesia for cataract surgery: patient perceptions of pain and side effects. Ophthalmology 2000; 107: 2054-2060.

4 Nijkamp MD, Ruiter RAC, Roelin M, van den Borne B, Hiddema F, Hendrikse $\mathrm{F}$ et al. Factors related to fear in patients undergoing cataract surgery: a qualitative study focusing factors associated with fear and reassurance among patients who need to undergo cataract surgery. Patient Educ Couns 2002; 47: 265-272.

5 Sumich PM, Francis IC, Kappagoda MB, Alexander SL. Artist's impression of endocapsular phacoemulsification surgery. J Cataract Refract Surg 1998; 24: 1525-1528.

6 Leo SW, Eong KG. Comments on anesthesia for cataract surgery. J Cataract Refract Surg 2003; 29: 633-634.

7 Leaming DV. Practice styles and preferences of ASCRS members - 2003 survey. J Cataract Refract Surg 2004; 30: 892-900. 
8 Ng DT, Rowe NA, Francis IC, Kappagoda MB, Haylen MJ, Schumacher RS et al. Intraoperative complications of 1000 phacoemulsification procedures: a prospective study. J Cataract Refract Surg 1998; 24: 1390-1395.

9 Francis IC, Schumacher RS, Haylen MJ. Assisted local anesthesia for cataract surgery (ALACS). Aust NZ J Ophthalmol 1987; 15: 185-191.

10 Lo Martire N, Savastano S, Rossini L, Pinchera L, Caracciolo F, Savastano MC et al. Topical anesthesia for cataract surgery with phacoemulsification: lidocaine $2 \%$ vs ropivacaine $1 \%$. Preliminary results. Minerva Anestesiol 2002; 68: 529-535.

11 Foggitt PS. Anxiety in cataract surgery: pilot study. J Cataract Refract Surg 2001; 27: 1651-1655.

12 Habib NE, Mandour NM, Balmer HG. Effect of midazolam on anxiety level and pain perception in cataract surgery with topical anesthesia. J Cataract Refract Surg 2004; 30: 437-443.

13 Fagerstrom R. Fear of a cataract operation in aged persons. Psychol Rep 1993; 72: 1339-1346.

\section{Appendix 1}

\section{Patient perception and experience in cataract surgery}

Thank you for completing this short survey. We would like to know how you found your surgery today, particularly what you remember and how you felt about the surgery. This will help us to understand the effects of sedation and how to improve care for future patients.

Please rate your pain by circling the one number that best describes your pain during the operation

$$
\begin{aligned}
& \begin{array}{lllllllllll}
0 & 1 & 2 & 3 & 4 & 5 & 6 & 7 & 8 & 9 & 10
\end{array} \\
& \text { No pain Pain as bad as you can imagine }
\end{aligned}
$$

Please rate your anxiety by circling the one number that best describes your anxiety during the operation

$\begin{array}{lllllllllll}0 & 1 & 2 & 3 & 4 & 5 & 6 & 7 & 8 & 9 & 10 \\ \text { No anxiety } & & & & & & & & & & \text { Anxiety as bad as you can imagine }\end{array}$

Please rate your fear by circling the one number that best describes your fear during the operation

$\begin{array}{lllllllllll}0 & 1 & 2 & 3 & 4 & 5 & 6 & 7 & 8 & 9 & 10 \\ \text { No fear } & & & & & & & & & \end{array}$

No fear $\quad$ Fear as bad as you can imagine

What did you recall during the operation? (tick all that apply)

$\begin{array}{lllll}\text { Colours } & \square & \text { Music } & \square & \text { Did not recall anything } \\ \text { Flashes } & \square & \text { Talking } & \square & \text { Other (please specify) } \\ \text { Light } & \square & \text { Equipment noises } & \square & \text {. }\end{array}$

Uncomfortably bright light

Shapes 